
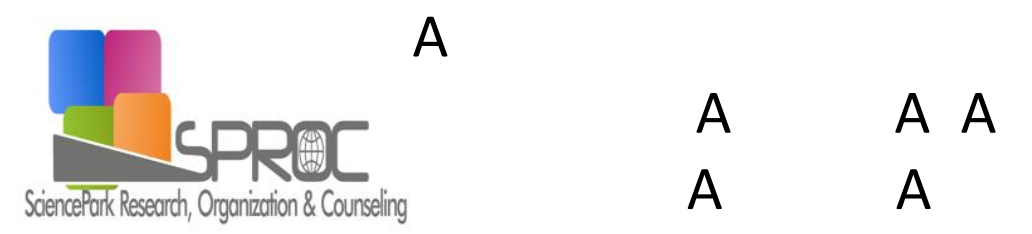

A A A A A A

www.gjsoc.eu

\title{
Trust in judiciary: a particular survey on Turkey
}

A

$\begin{aligned} & \text { Erhan Orselli* A } \\ & \text { Esra Banu Sipahi }\end{aligned}$
A

Abstract

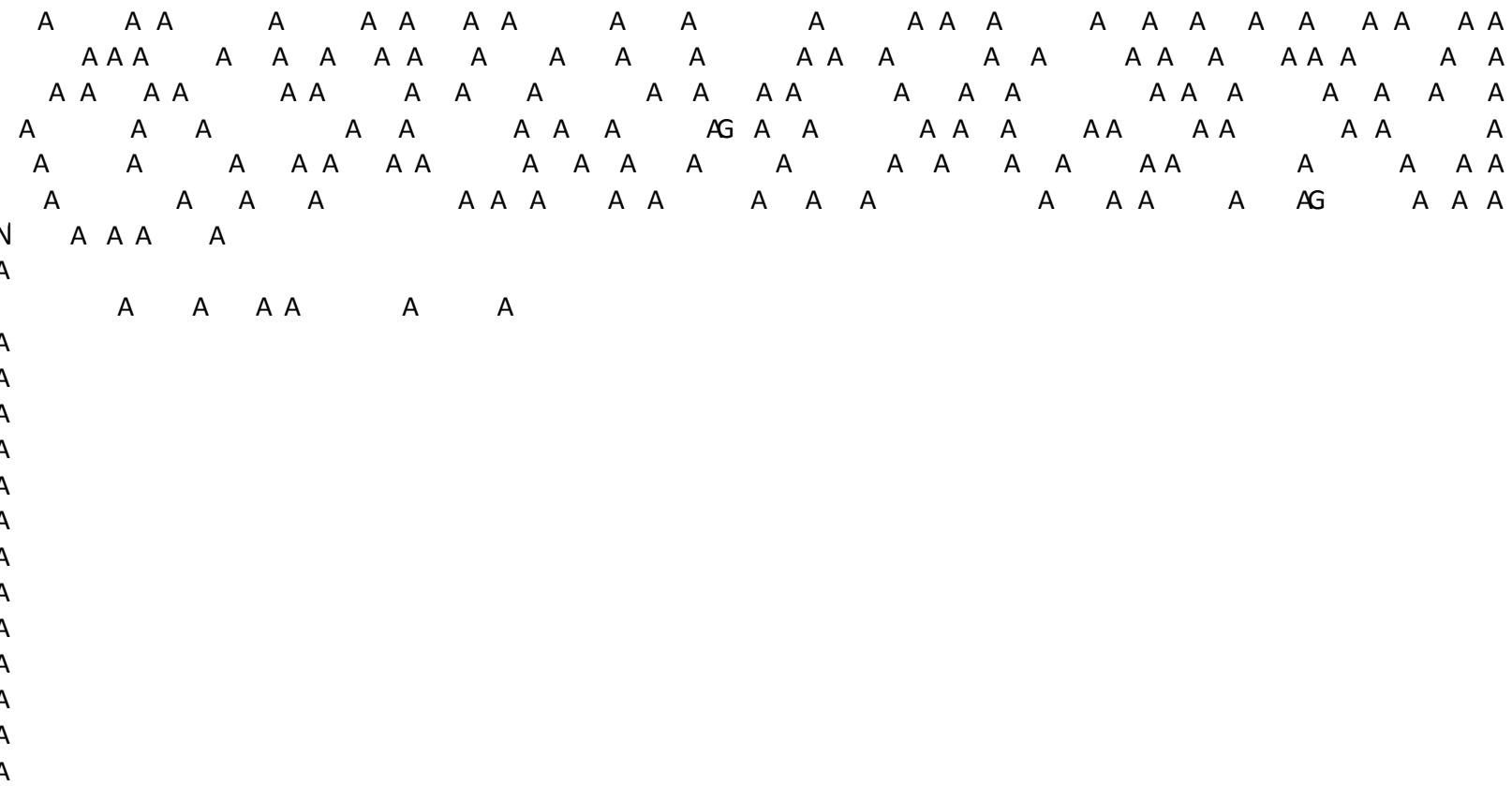

$\begin{array}{rrrrrrrrrrrrrrrrrr}* & & \text { A } & \text { A } & & & \text { Erhan Orselli,A } & \text { A A } & \text { A } & \text { A } & \text { A } & \text { A } & & \text { A } & \text { A A }\end{array}$
A 


\section{Introduction}

A

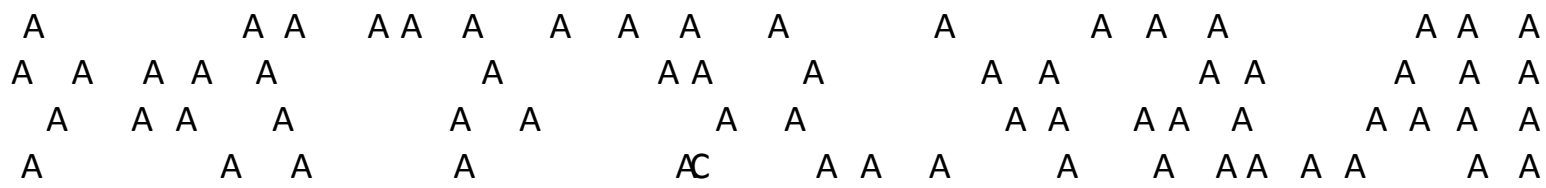
A A A A A A A A A A A A A A A A A A $\begin{array}{lllllllllllllllll}A & A & A & A & A & A & A & A & A & A & A & A & A & A & A\end{array}$ $\begin{array}{lllllll}A & A & A & A & A & A & A\end{array}$

A A A A A A A A A A A A A A A A A A A AA

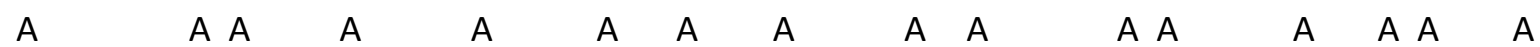
$\begin{array}{lllllllllll}A & A & A & A & A & A & A & A & A & A & A\end{array}$ A A A A A A A A A A

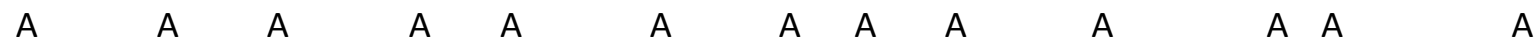
$\begin{array}{llllllllllllllllllll}A & A & A & A & A & A & A & A & A & A & A & A & A & A & A & A & A\end{array}$

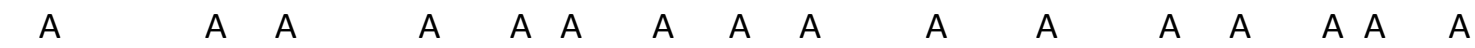

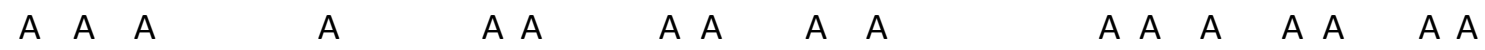
$A \quad A \quad A \quad A \quad A A$ A A A A A A A A $\begin{array}{lllllllllllllll}A & A & A & A & A & A & A & A & A & A & A & A & A & A\end{array}$

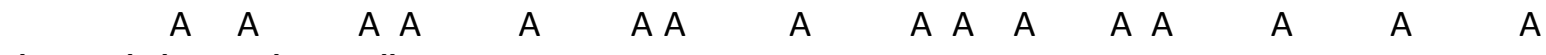
$A \quad A A \quad A \quad A$

$\begin{array}{lllllllllllllllll}A & A & A & A & A & A & A & A & A & A & A & A & A & A & A & A\end{array}$ $A \quad A \quad A A$ A A AAA A A A A A A A A A A A A $A \quad A \quad A A$ A $A A A$ A A A A A A A A A A A

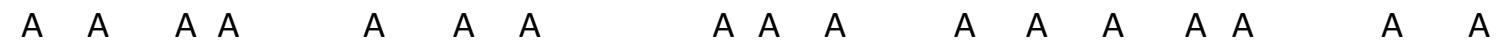
A A A A A A A A A A A A A A A A A A A A A A What is the citizens' the level of trust in judiciary? Which factors affect the citizens' trust in judiciary? What is the level of satisfaction from judicial services? A

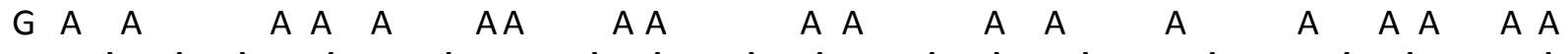
$\begin{array}{llllllllllllllllllllll}A & A & A & A & A & A & A & A & A & A & A & A & A & A & A & A\end{array}$

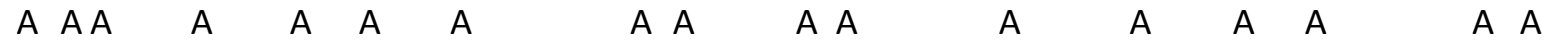

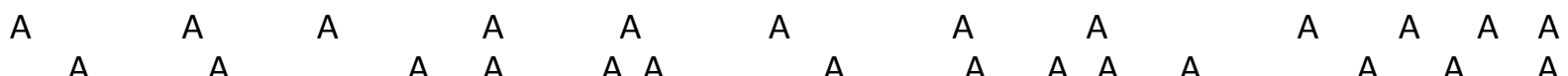

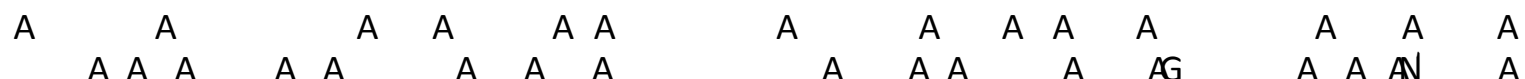

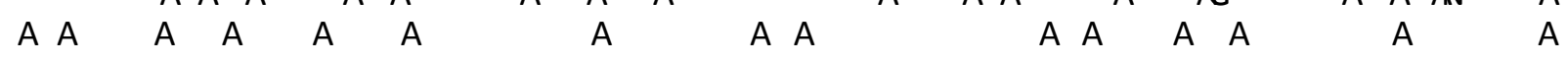

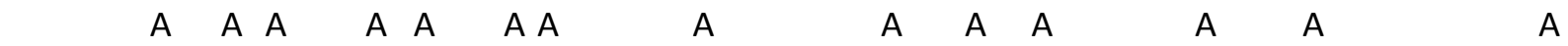
$\begin{array}{lllllllllllll}A & A & A & A & A & A & A & A & A & A & A & A & A\end{array}$ $\begin{array}{lllllllllllllllllll}A & A & A & A & A & A & A & A & A & A & A & A & A & A & A & A & A & A\end{array}$

\section{Trust and Trust in Judiciary}

A

$\begin{array}{llllllllllll}A & A & A A & A & A & A & A & A & A & A & A & A\end{array}$

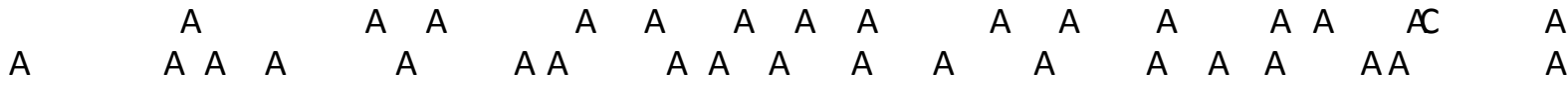

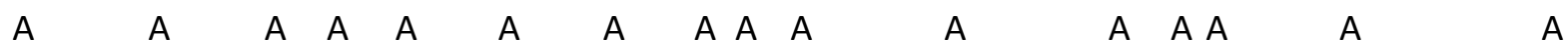
A A A A A A 


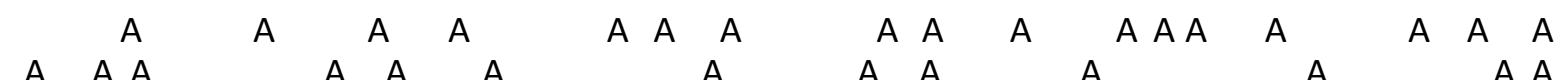

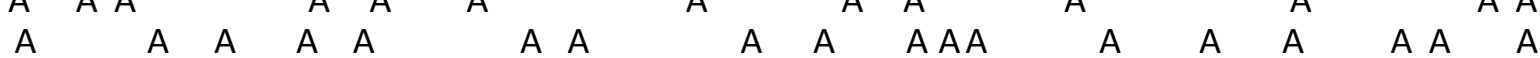

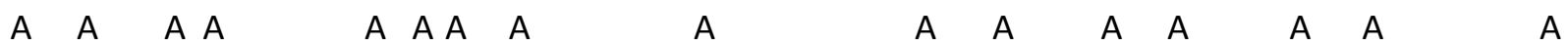

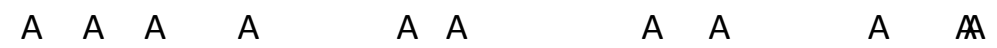

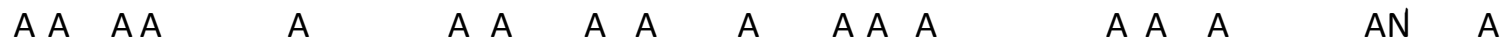
$\begin{array}{llllllllllllllllllll}A & A & A & A & A & A & A & A & A & A & A & A & A & A & A\end{array}$ $\begin{array}{llllllllllllll}A & A & A & A & A & A & A & A & A & A & A & A & A\end{array}$ $\begin{array}{llllllllllllllll}A & A & A & A & A & A & A A & A & A & A A & A & A & A & A\end{array}$

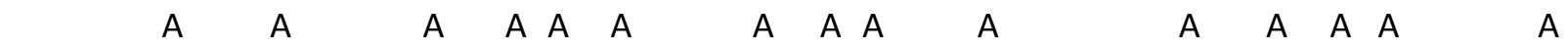

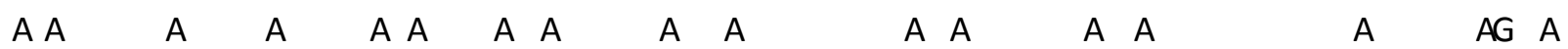
$\begin{array}{lllllllllllllllllllllll} & A & A & A & A & A & A & A & A & A & A & A & A & A & A & A & & A & A & A\end{array}$

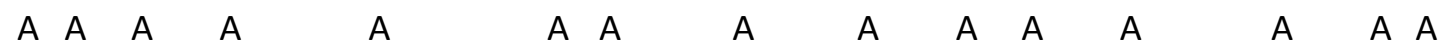

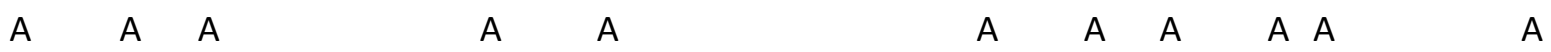

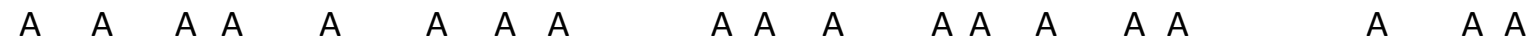

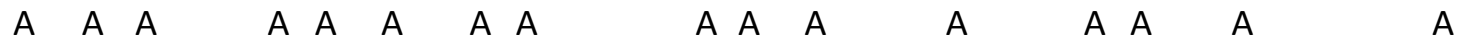
A $\quad A \quad A A$ A $A A$ A $A A$ A A $A$ A A A $A$ A A A A A A A A A A A A A A A A A A A A A $A \quad A$

$\begin{array}{lllllllllllllllll}A & A & A & A & A & A & A & A & A & A & A & A & A & A & A & A & A\end{array}$

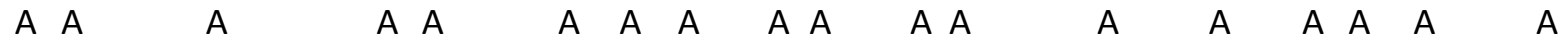

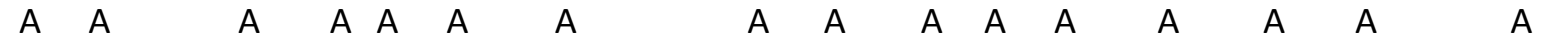
$\begin{array}{llllllllllllllllllllll}A & A & A & A & A & A & A & A & A & A & A & A & A & A\end{array}$ $\begin{array}{lllllllllllll}A & A & A & A & A & A & A & A & A & A & A & A & A\end{array}$

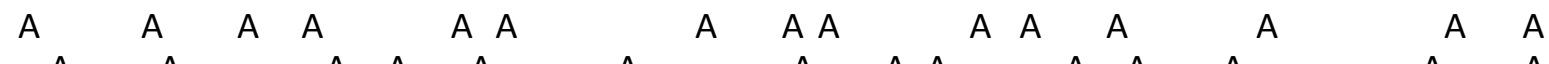
$\begin{array}{lllllllllllll}A & A & A & A & A & A & A & A & A & A & A & A & \end{array}$ A A A A A A A A A A $\quad$ A A A $A$ A $A$ A $A$ A

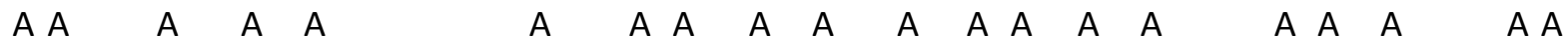

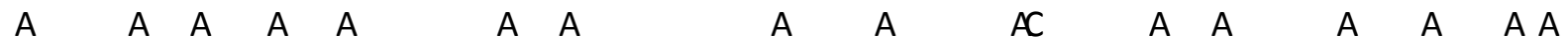
$\begin{array}{lllllll}A & A & A & A & A A & A & A\end{array}$

$\begin{array}{lllllllllllllllllll}A & A & A & A & A & A & A & A & A & A & A & A & A & A & A\end{array}$ $\begin{array}{lllllllllllll}A & A & A A & A & A & A & A A & A A & A & A & A & A & A\end{array}$

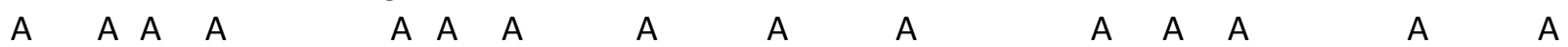
$A$ A $A A$ A $A$ A $A$ A A A A A A A A A A A

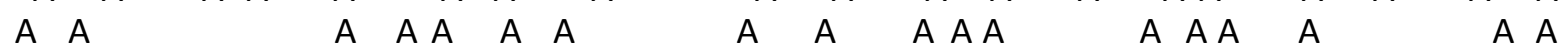
A A A A A $\quad$ A A

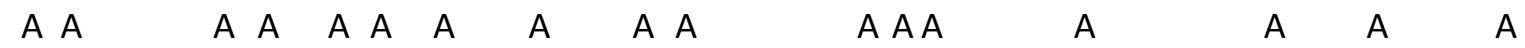

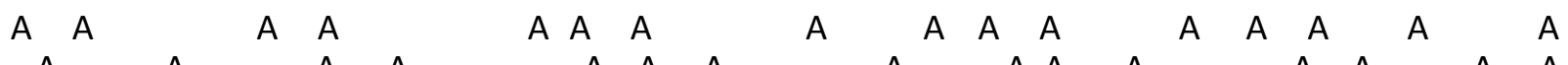
$\begin{array}{llllllllllllllllll}A & A & A & A & A & A & A & A & A & A & A & A & A & A & A\end{array}$ $A A \quad A C \quad A \quad A A \quad A \quad A$

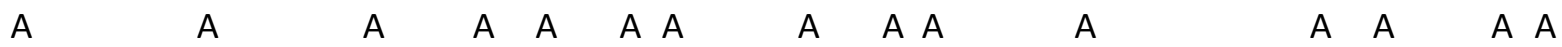

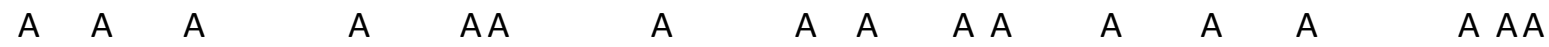
$\begin{array}{llllllllllllll}A & A & A & A & A & A & A & A & A & A & A & A & A & A\end{array}$ A A Asine qua nonA A $\quad$ A $\quad$ A A A A A A A A $A$ A the principle of democratic, secular and social state ruled by law A A A 
A

A $A$ A A A A

A $A \quad A \quad A \quad A$ $\begin{array}{lllllllll}A & A & A & A & A & A & A & A & A\end{array}$
A A $A A A$ A A A A A $A$ A A A $A$ A $\quad A \quad A$
A A A
$A$

A A A

$A A \quad A \quad A \quad A$

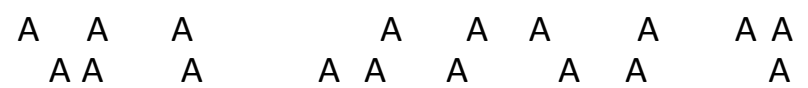

A A

A A A

A A

A A $A$ A A A A A A

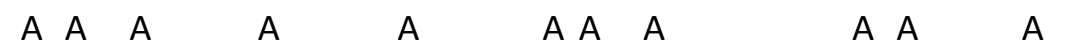

A A A A A A A A A A A A A A

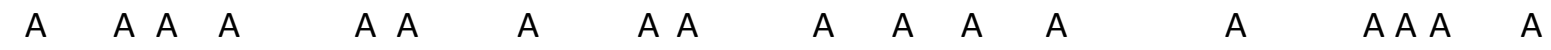

$A$ A A A A A A A A A A A A A A A A A A A

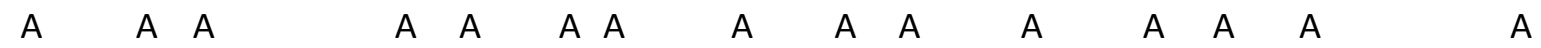
$\begin{array}{llllllllllllllll}A & A & A & A & A & A & A & A & A & A & A & A & A & A & A & A\end{array}$ $\begin{array}{llllllllllllllllllllllllllllll}A & A & A & A & A & A & A & A & A & A & A & A & A & A & A & A & A & A & & A\end{array}$ A A A A A A A A A

\section{Aim and Methodology of the Study}

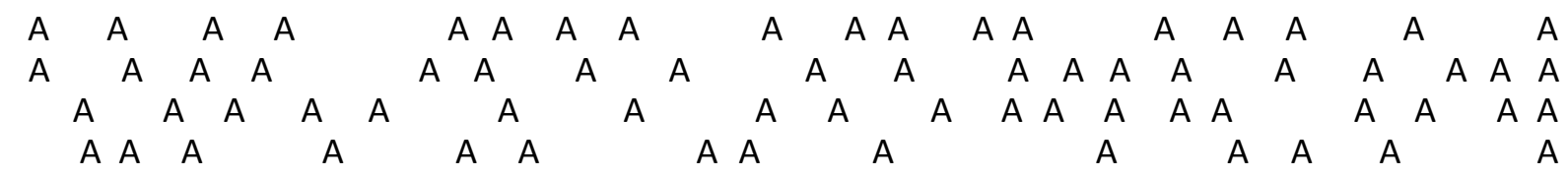
$A \quad A \quad A A \quad A$

A A A A A A A Arima Research Consultation and Education Services Limited Company A A A A A A A A A A AG A A AN A A A A A $\begin{array}{lllllllllllllllllllll}A & A & A & A & A & A & A & A & A & A & A & A & A & A & A & A\end{array}$ $\begin{array}{lllllllllllllll}A & A & A & A & A & A & A & A & A & A & A & A & A & A\end{array}$ A

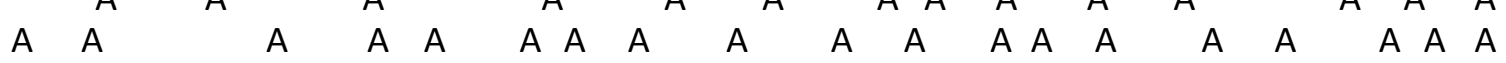

$$
\begin{array}{llllllllllll}
A & A & A & A & A & A & A & A & A & A & A & A
\end{array}
$$

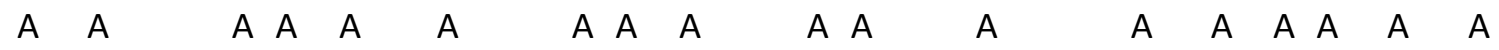
A A A A A A A A A A A A A A A A A A $A$ A A A A A A A A A A Nomenclature of Units for Territorial Statistics

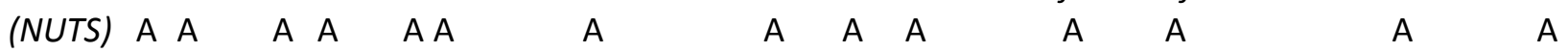

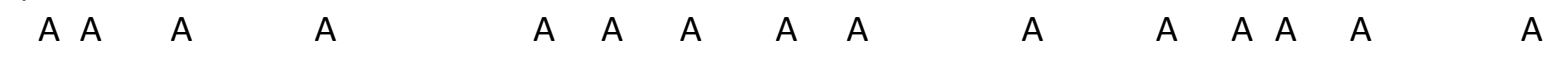
A A A A A A A A A A

\section{Findings of the Study}

\subsection{Analysis of the Socio-Demographical Characteristics of the Participants}

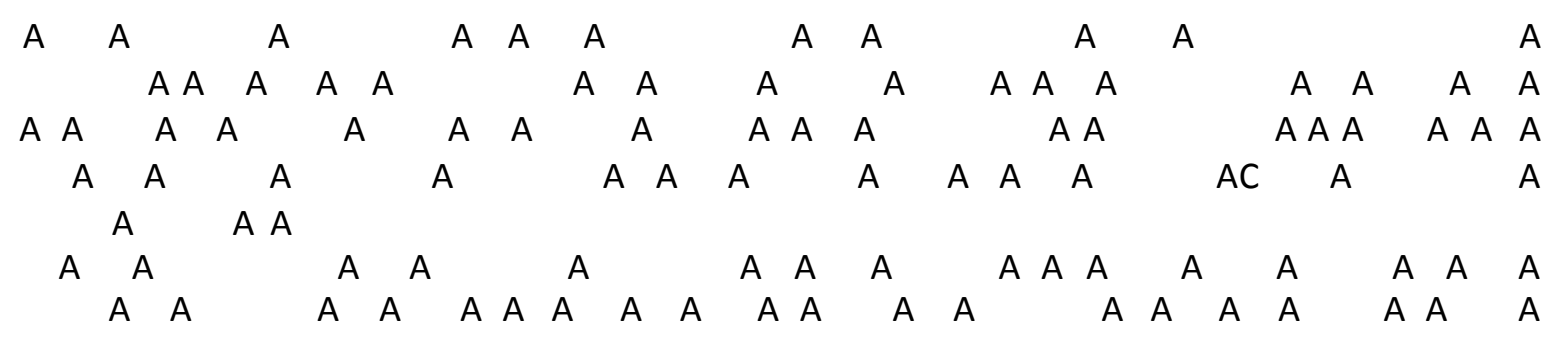


$A \quad A \quad A A \quad A \quad A \quad A \quad A \quad A \quad A A \quad A \quad A \quad A \quad A \quad A A A A$ A A $\begin{array}{lllllllllllllllllllllll}A & A & A & A & A & A A & A & A & A & A & A & A & A & A & A\end{array}$

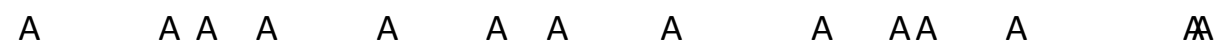

$\begin{array}{lllllllllllllllll}A & A & A & A & A & A & A & A & A & A & A & A & A & A & A & A & A\end{array}$

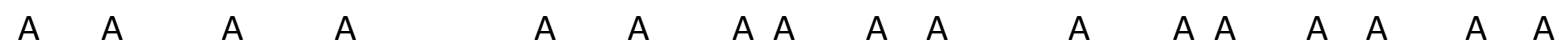

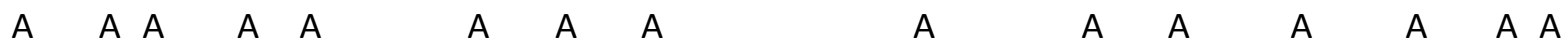
A A A A A A A

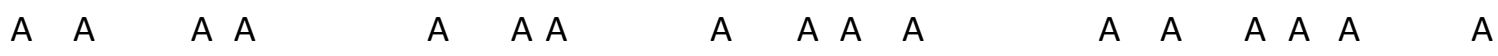
$\begin{array}{llllllllllllllll}A & A & A & A & A & A & A & A & A & A & A & A & A & A & A & A\end{array}$ $\begin{array}{llllllllllllllll}A & A & A & A & A & A & A & A & A & A & A & A & A & A\end{array}$ $\begin{array}{lllllllllllllll}A & A & A & A & A & A & A & A & A & A & A & A & A & A\end{array}$ A A A A A A A A A A A

G $A$ A A A A A A $\begin{array}{lllllllllllllllllll}A & A & A & A & A & A & A & A & A & A\end{array}$

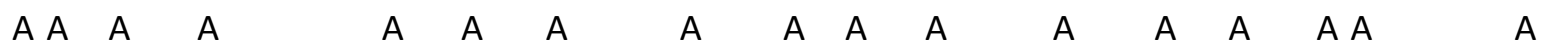

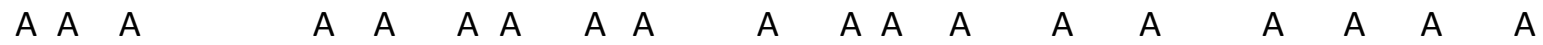
$\begin{array}{llllllllllllllllll}A & A & A & A & A & A & A & A & A & A & A & A & A & A & A & A & A & A\end{array}$ $\begin{array}{llllllllllllllllllll}A & A & A & A & A & A & A & A & A & A & A & A & A & A & A & A & A & A & A & A\end{array}$ A A A A A A A A A

$\begin{array}{lllllllllllllll}A & A & A & A & A & A & A & A & A & A & A & A & A & A & A\end{array}$ $\begin{array}{lllllllllllll}A & A & A & A & A & A & A & A & A & A & A & A & A\end{array}$ $\begin{array}{llllllllllll}A & A & A & A & A & A & A & A & A & A & A & A\end{array}$

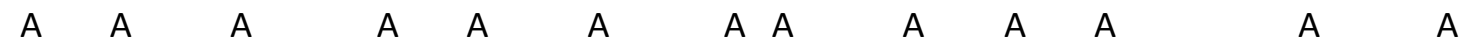

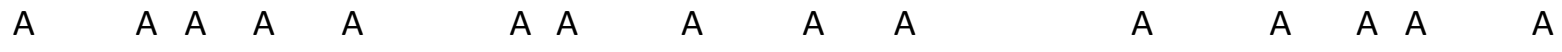
$\begin{array}{llllllllllllll}A & A & A & A & A & A & A & A & A & A & A & A & A & A\end{array}$ $\begin{array}{llllllllllllllll}A & A & A & A & A & A & A & A & A & A & A & A & A & A & A\end{array}$ $\begin{array}{llllllllllll}A & A & A & A & A & A & A & A & A & A & A & A\end{array}$ A A A A

\subsection{Citizens' Trust in Judiciary in Turkey}

$\begin{array}{lllllllllllllllllllllllllllll}A & A & A & A & A & A & A & A & A & A & A & A & A & A & A & A & A\end{array}$

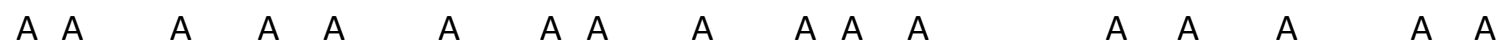
$\begin{array}{llllllllllllll}A & A & A & A & A & A & A & A & A & A & A & A & A & A\end{array}$ A A

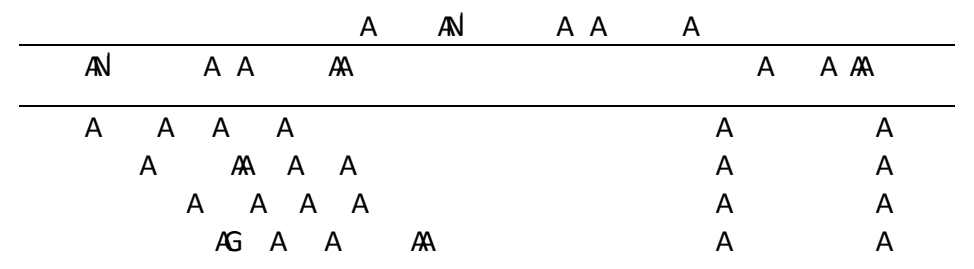

\begin{tabular}{llllllllllll} 
& & \multicolumn{3}{c}{ A A } & A & \multicolumn{1}{c}{ A } & & A & A \\
\cline { 2 - 7 } & A & A A A & A & A & A & A & A A & A & A A A & A A
\end{tabular}

A

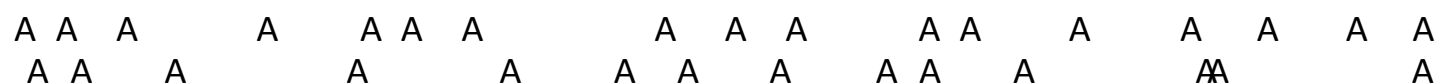

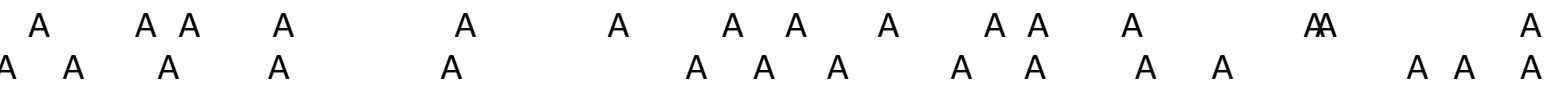

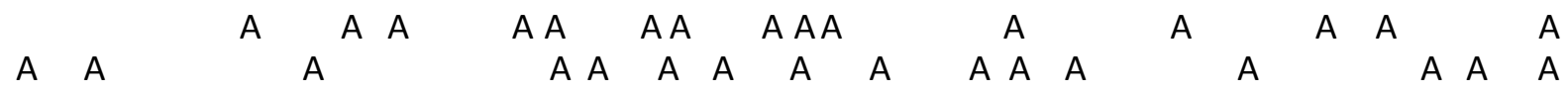


$\begin{array}{llllllllllllllll}A & A & A & A & A & A & A & A & A & A & & A & A & A & A & A\end{array}$

A

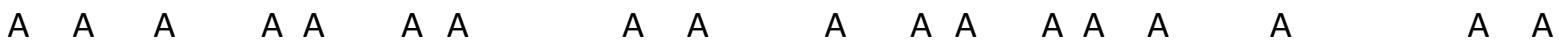
$\begin{array}{lllllllllllllllllllllllllllll}A & A & A & A & A & A & A & A & A & A & A & A & A & A & A & A & A & A\end{array}$ $A \quad A \quad A \quad A \quad A \quad A \quad A A A A$ A A A A A A A A

$\begin{array}{lllllllllll}A & A & A & A & A & A & A & A & A A & A & A\end{array}$ $\begin{array}{llllllllllllllllllllllllllll}A & A & A & A & A & A & A & A & A & A & A & A & A C & A & A & A\end{array}$ $\begin{array}{llllllllllll}A & A & A & A & A & A & A & A & A & A & A & A\end{array}$ $\begin{array}{llllllllllllllllll}A & A & A & A & A & A & A & A & A & A & A & A & A & A & A & \end{array}$

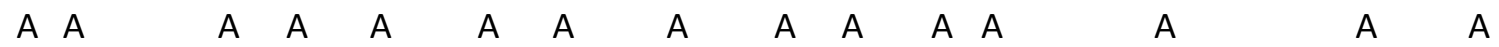

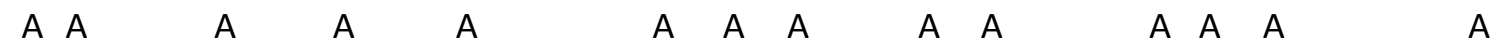
$\begin{array}{llllllllllllll}A & A & A & A & A & A & A & A & A & A & A & A & A & A\end{array}$ A A A A

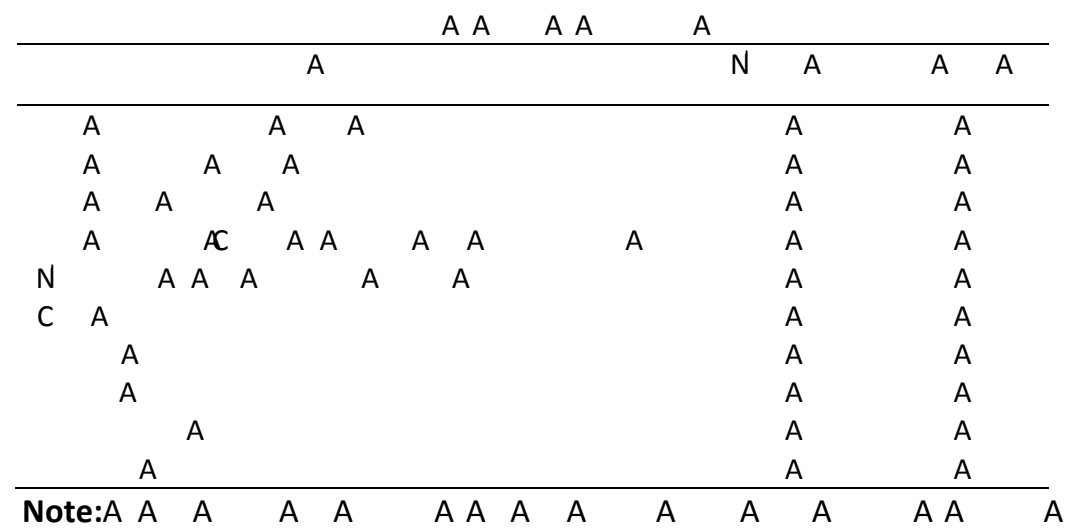

$A$ A A A A

C A A A A A A A
A $A$
$\begin{array}{llllllll}A & A & A & A & A & A & A & A\end{array}$

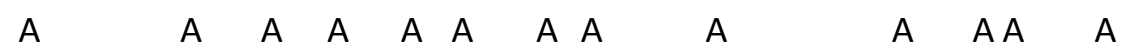
$\begin{array}{lllllllll}A & A & A & A & A & A & A & A & A\end{array}$

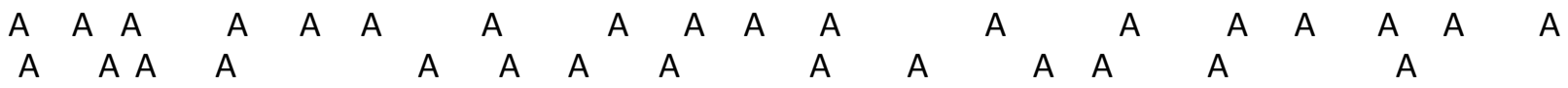
$\begin{array}{llllllllllllllll}A & A & A & A & A & A & A & A & A & A & A & A & A & A & A & A\end{array}$ $\begin{array}{llllllllllllllllllllll}A & A & A & A & A & A & A & A & A & A & A & A & A & A & A & A & A & A & A\end{array}$ $\begin{array}{llllllllllllllllllllll}A & A & A & A & A & A & A & A & A & A & A & A & A & A & A & A & A & A\end{array}$

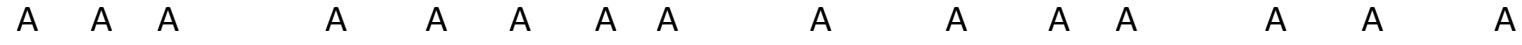
$\begin{array}{lllllllllllllllll}A & A & A & A & A & A & A & A & A & A & A & A & A & & A & A\end{array}$

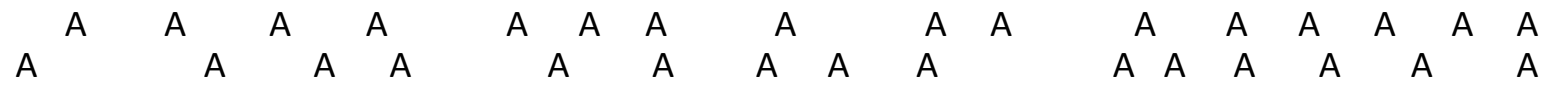
$A A \quad A A \quad A \quad A$

$\begin{array}{llllllllll}A & A & A A & A & A & A & A & A & A & A\end{array}$ $\begin{array}{llllllllllllll}A & A & A & A & A & A & A & A & A & A & A & A & A & A\end{array}$

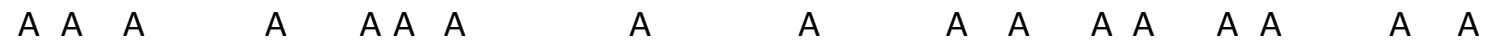
$\begin{array}{lllllllllllllllll}A & A & A & A & A & A & A & A & A & A & A & A & A & A & A & A\end{array}$ $\begin{array}{lllllllllll}A & A & A & A C & A & A & A & A & A & A & A\end{array}$ $\begin{array}{llllllllllllllll}A & A & A & A & A & A & A & A & A & A & A & A A & A & A & A & A\end{array}$

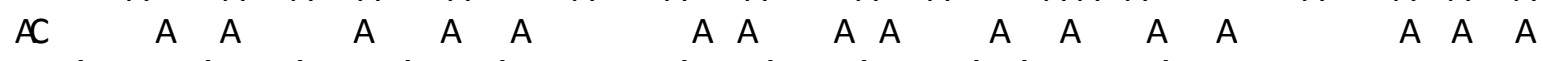




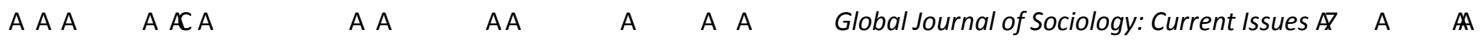

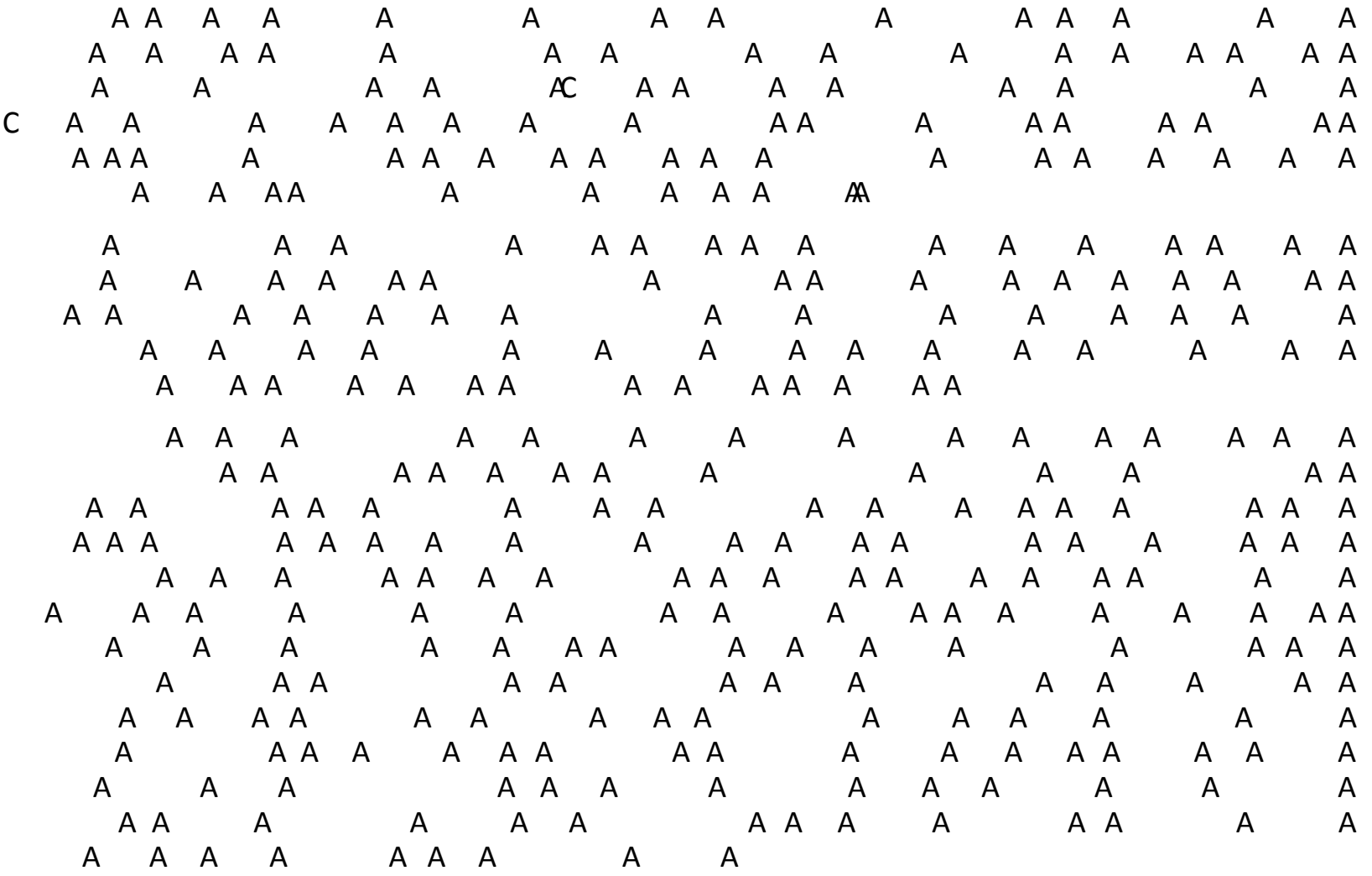

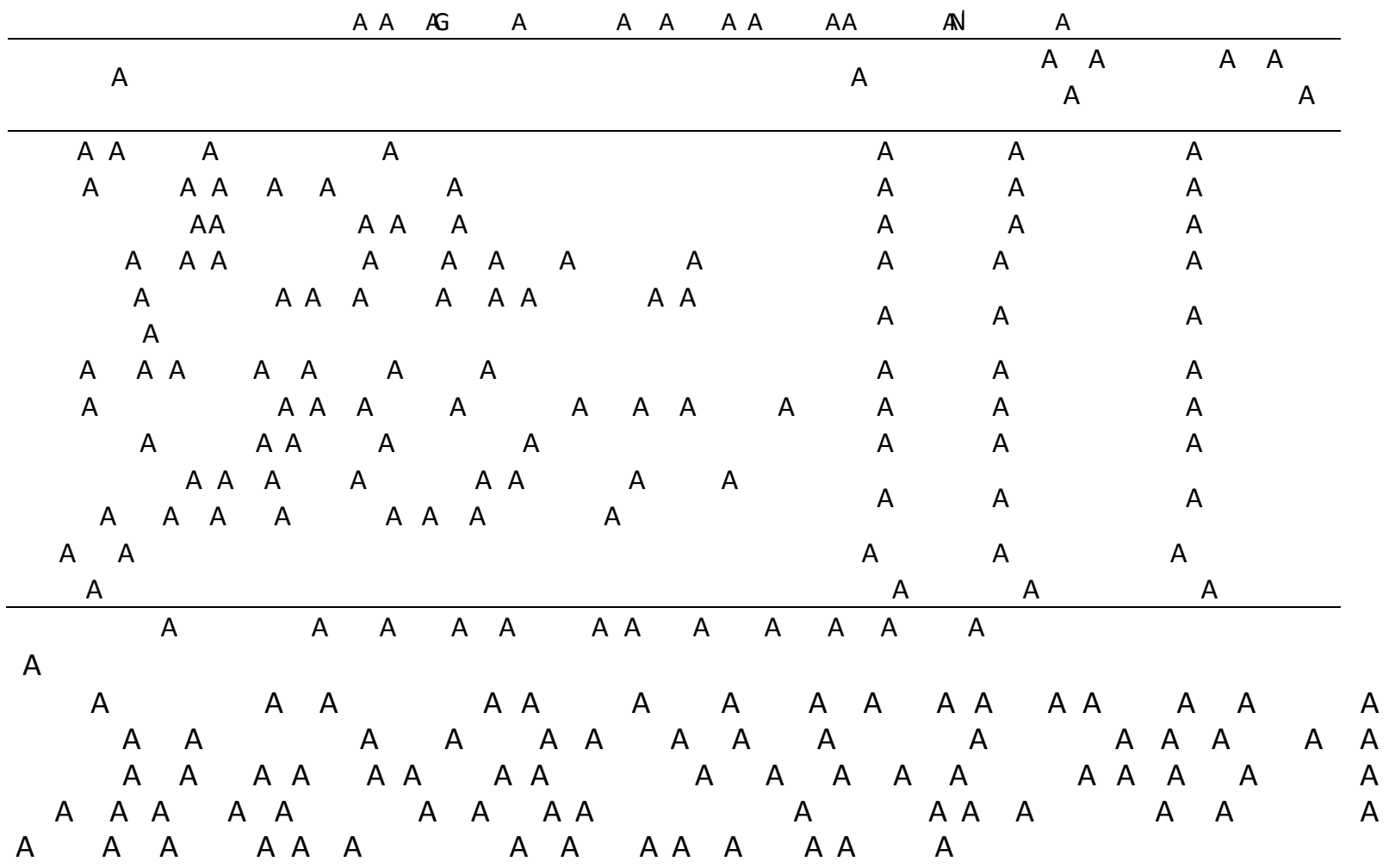

50 


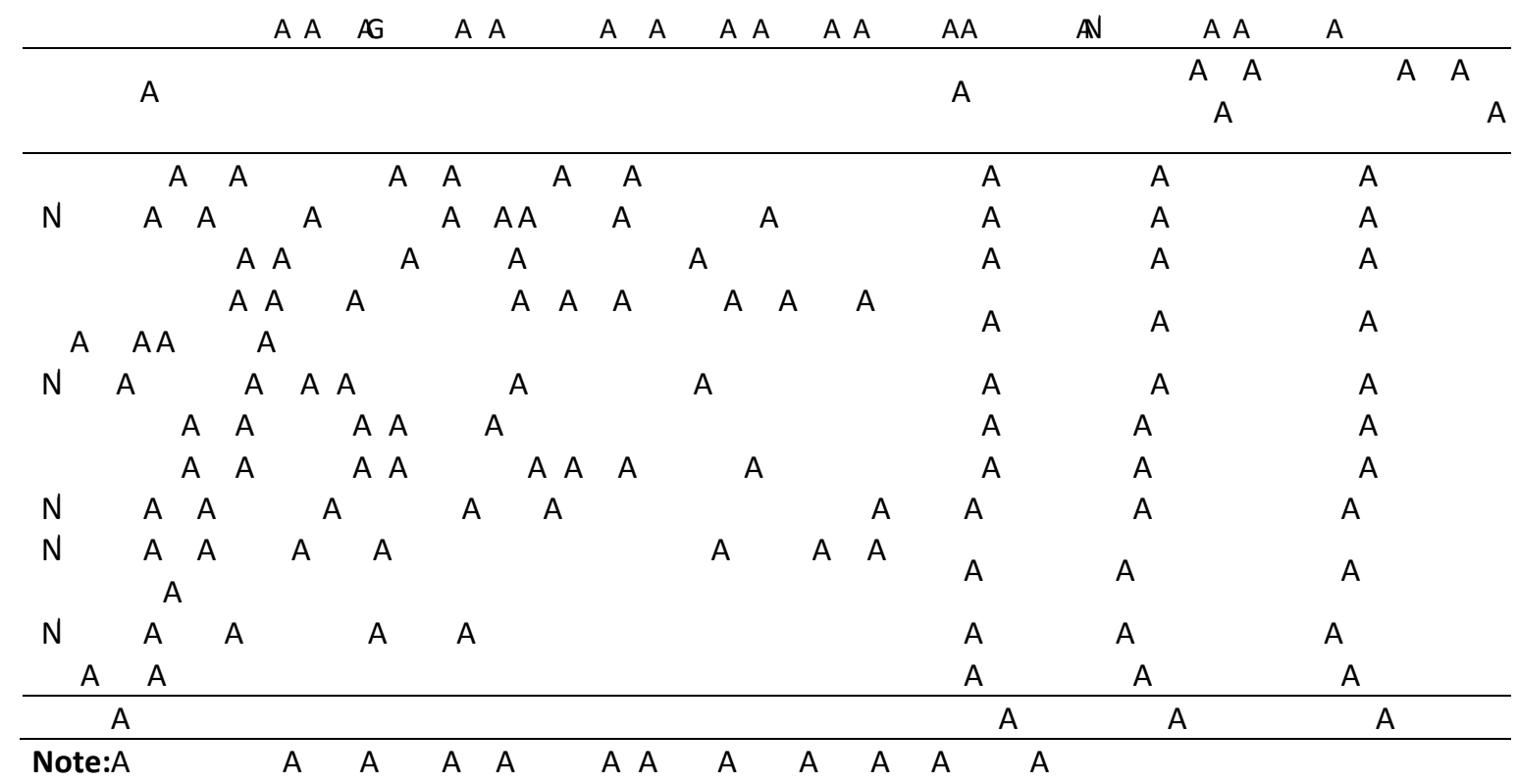

A

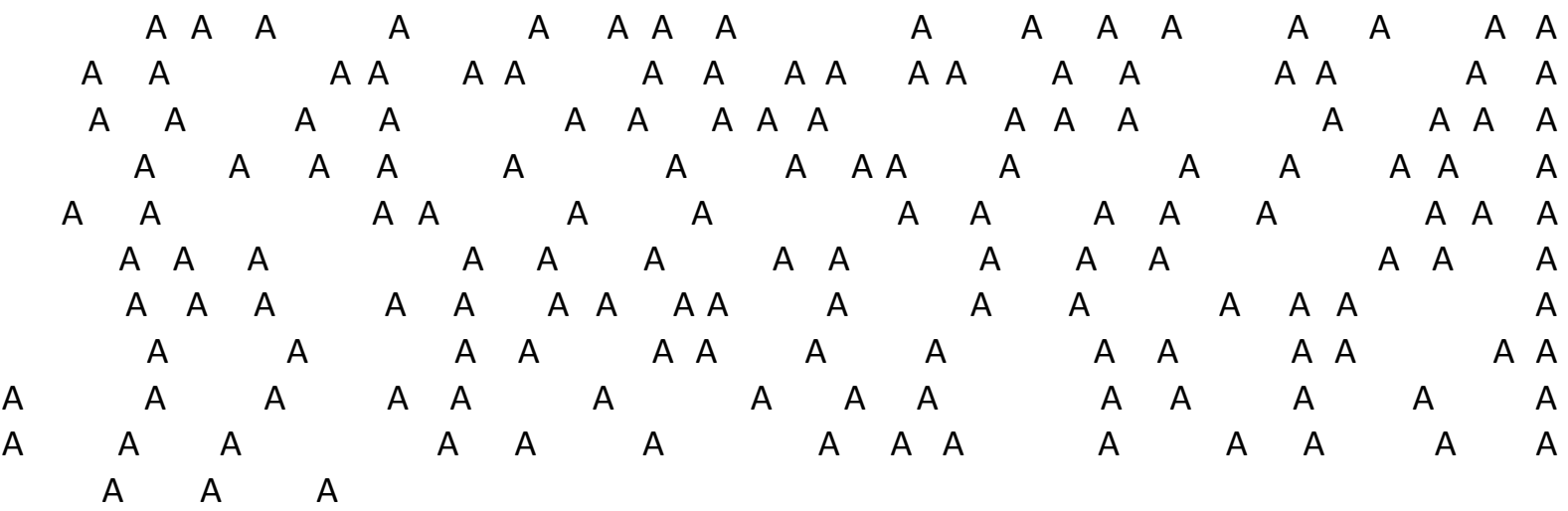

\section{Conclusion}

A

$\begin{array}{llllllllllllllll}A & A & A & A & A & A & A & A & A & A & A & A & A & A & A\end{array}$ $\begin{array}{llllllllllllllllllll}A & A & A & A & A & A & A & A & A & A & A & A & A & A\end{array}$ $\begin{array}{llllllllllll}A & A & A & A & A & A & A A & A A & A & A & A & A\end{array}$

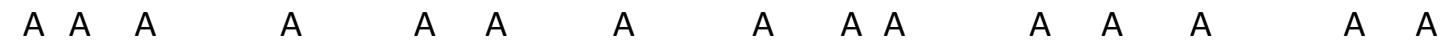
$A A$ A A A A A A A A A

$\begin{array}{lllllllllllllllllll}A & A & A & A & A & A & A & A & A & A & A & A & A & A & A & A & A & A & A\end{array}$

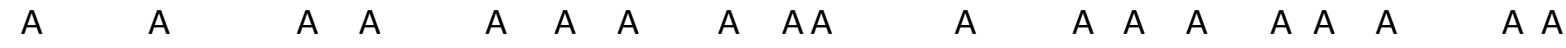
$A \quad A \quad A \quad A A$ A $A$ A A A A A A A A A A A A A

A A A A A Athe State and laws A A A Aequality before the law A compliance with the law A distinguishing fair and unfair AA A protection of the weak/oppressed A A A A A A A

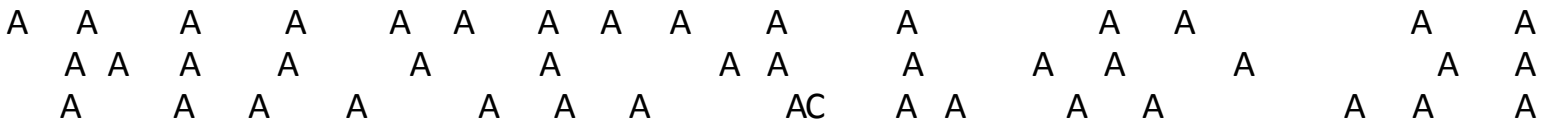




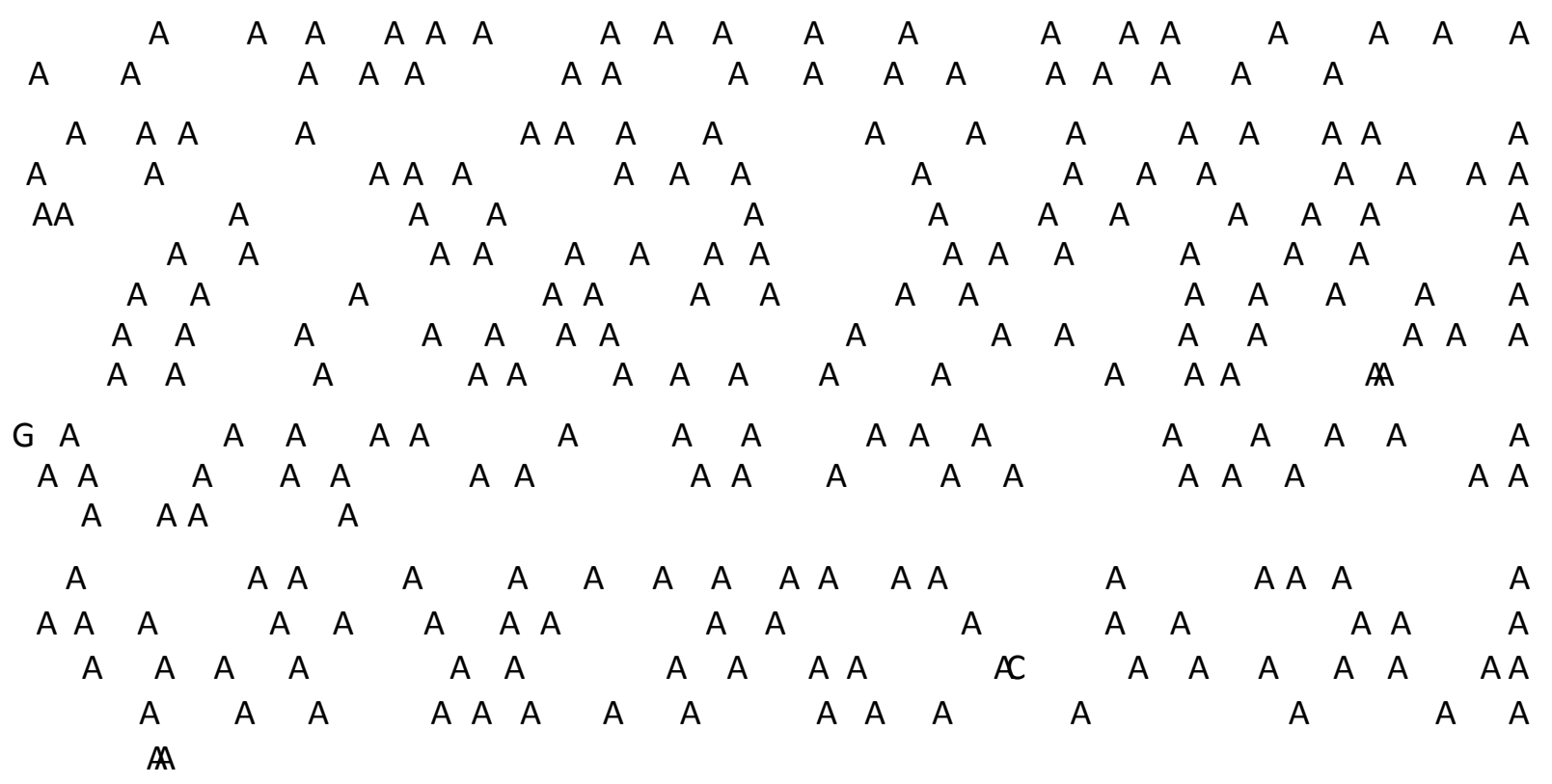

\section{References}

A

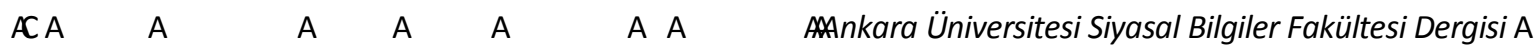
$56 \mathrm{~A} \quad \mathrm{~A}$

C A A A A A A A
A A A
A A
A A A A
ABehavioral

Sciences and The Law $19 \mathrm{~A} \quad \mathrm{~A}$

C AN A A A A A

AGA A ATurkiye'de yargiya toplumsal bakis A AC N A

A A A A A A A

A A

A A A A A

A A A ATrust

in Government: Innovations in Governance Reform in Asia A A A A A A A A A

AGA A A A A AGA A ASosyal Bilimlerde GüvenA A $\quad$ A $\quad$ A A A A A A A A A A A Arust: Making and Breaking Cooperative Relations A A A A $A \subset A C$

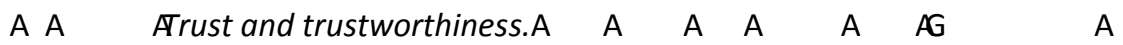

A A A A A A $\quad$ A $A$ A $A$ A A $A$ A A A A A A Adalet Gözet, Yargı Sistemi Üzerine Bir Inceleme A A $\quad$ A $\quad$ A $\quad$ A $\quad$ A $\quad$ A $\quad$ A A A A A A A A Adalet barometresi; vatandaslarin mahkemeler hakkindaki gorusleri ve degerlendirmeleri A A $\quad A \quad A \quad A \quad A \quad A$

AN A A A A A AGA A ASosyal Bilimlerde Güven A $\quad$ A $\quad$ A A

A A A A A A A AA A Aocial Forces $163 \mathrm{~A}$ A
A A
AG
A
A A

Making and Breaking Cooperative Relations A

A A A A

N A A A

A A

A

A A A A A

$\begin{array}{lllllllllll}\text { A A A } & \text { A ACA } & \text { A } & \text { A A } & \text { A } & \text { A A } & \text { A } & \text { A } & \text { A } & \text { A5th International Conferenc } \\ \text { Research in Humanities, Sociology and Corporete Social Responsibility } & \mathbb{N} & \text { A } & \text { A A A }\end{array}$

$\begin{array}{lllllllll}\text { A A A A ACA A A A A } & \text { A A A } & \text { A } & \text { A } & \text { A5th International Conference on } \\ \text { Research in Humanities, Sociology and Corporete Social Responsibility } & \mathbb{N} & \text { A } & \text { A A } & \text { A }\end{array}$

A A Aürkiye'de yargıya güven $\mathrm{A} \quad \mathbb{D} \mathrm{A} \quad \mathrm{A}$
A A A
A A A
AAmerica at risk the crisis of hope, trust, and caring. A A
A
A A 
A A AMaking democracy work A $\quad$ A $\quad$ A $\quad$ A $A$ AN A A A A A A A Political Science Quarterly, 115, A $\quad$ A

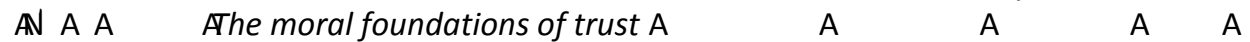
AN A A A A A A A A A A A A A A A A A A A AHook of Social Capital A A A A

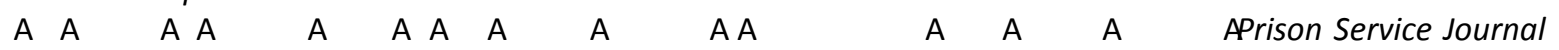
183, A A 